\title{
Bosentan Effects on Hemodynamics and Clinical Outcome in Heart Failure Patients with Pulmonary Hypertension Awaiting Cardiac Transplantation*
}

\author{
T. Hefke ${ }^{1, * *}$ \\ A. Zittermann ${ }^{1, * *}$ \\ U. Fuchs 1 \\ S. Schulte-Eistrup ${ }^{1}$ \\ J. F. Gummert ${ }^{1}$ \\ U. Schulz ${ }^{1}$
}

\author{
${ }^{1}$ Department of Cardio-Thoracic Surgery, Heart Center NRW, Bad \\ Oeynhausen, Germany \\ **The first two authors contributed equally to this work.
}

\begin{abstract}
Address for correspondence and reprint requests Uwe Schulz, Department of Cardio-Thoracic Surgery, Heart Center NRW, Georgstraße 11, 32545 Bad Oeynhausen, Germany (e-mail: uschulz@hdz-nrw.de).
\end{abstract}

Thorac Cardiovasc Surg 2012;60:26-34.

\begin{abstract}
Background In heart failure (HF) patients, pulmonary hypertension (PH) is associated with a poor prognosis. We assessed whether low dose treatment with the dual endothelin-1 receptor antagonist bosentan is associated with improved hemodynamics and clinical outcome in these patients.

Methods We performed a retrospective data analysis in 82 end-stage heart failure patients on the waiting list for cardiac transplantation since January 2006. All patients had pulmonary arterial pressure $>35 \mathrm{mmHg}$, pulmonary vascular resistance $>240$ dyn $\times \mathrm{s} \times \mathrm{cm}^{-5}$, and/or a transpulmonary gradient (TPG) $>15 \mathrm{mmHg}$. Fifty-four patients received a median dose of $125 \mathrm{mg}$ bid bosentan (BOS group), and 28 patients received standard medical treatment (CON group). Data were assessed until June 2009. Results Hemodynamic parameters improved significantly in the BOS group but remained unchanged in the CON group. The percentage of patients who fell below the thresholds of PAP, PVR, and TPG for cardiac transplantation increased significantly by

Keywords

- end-stage heart failure

- bosentan

- pulmonary hypertension

- cardiac transplantation

- mortality

- survival

- endothelin-1 $20.3 \%, 34.5 \%$, and $20.8 \%$, respectively ( $p=0.007-0.013)$ in the BOS group, but did not change significantly in the CON group. One-year survival on the waiting list was approximately $20 \%$ higher in the BOS group than in the CON group $(p=0.020)$. Bosentan treatment remained an independent predictor of reduced mortality risk on the waiting list after propensity score adjustment (relative risk $=0.107 ; 95 \% \mathrm{Cl}: 0.013-$ $0.869 ; p=0.036)$.

Conclusion Treatment with the endothelin-1 antagonist bosentan is associated with improvements in hemodynamics and clinical outcome in end-stage heart failure patients with $\mathrm{PH}$. If these results can be confirmed by randomized controlled trials, bosentan may represent a treatment option in these patients.
\end{abstract}

\section{Introduction}

Pulmonary hypertension $(\mathrm{PH})$ is a pathophysiological condition defined as an increase in mean pulmonary arterial

\footnotetext{
*This work was presented at the 5th Joint Meeting: German, Austrian and Swiss Society for Thoracic and Cardiovascular Surgery, Innsbruck, February 17th to 20th, 2008.
}

pressure (PAP) $\leq 25 \mathrm{mmHg}$ at rest. ${ }^{1}$ PH due to left heart failure is associated with increased pulmonary vascular resistance (PVR) and elevated transpulmonary pressure gradient (TPG). ${ }^{2}$ In addition, it carries a poor prognosis. Mortality rates of $57 \%$ in patients with moderate $\mathrm{PH}$ compared with $17 \%$ in patients without $\mathrm{PH}$ have been reported after 28 months of follow-up. ${ }^{3}$ Survival rates are lower in patients with received

October 13, 2010

published online

March 22, 2011
Copyright $\odot 2012$ by Thieme Medical Publishers, Inc., 333 Seventh Avenue, New York, NY 10001, USA. Tel: +1(212) 584-4662.
DOI http://dx.doi.org/ 10.1055/s-0030-1250726. ISSN 0171-6425. 
pulmonary hypertension and poor right ventricular (RV) function compared to patients with elevated PAP but preserved RV function. ${ }^{4}$

In end-stage heart failure patients, preoperatively existing $\mathrm{PH}$ is associated with a high risk of right heart failure and mortality after cardiac transplantation. ${ }^{5,6,7}$ According to the International Society for Heart and Lung Transplantation and the German Standing Committee on Organ Transplantation, cardiac transplantation is contraindicated as long as values of PAP, PVR, and/or TPG are above $40 \mathrm{mmHg}, 240$ dyn $\times \mathrm{s}$ $\times \mathrm{cm}^{-5}$ and 15 , respectively. ${ }^{5,8}$ At present, no specific therapy for $\mathrm{PH}$ due to left heart disease is available. ${ }^{1}$

The endothelin A and B receptor antagonist bosentan has been shown to improve pulmonary and systemic hemodynamics in patients with heart failure. ${ }^{7,9,10}$ The plasma concentration of endothelin-1 (ET-1) is elevated in chronic heart failure patients and is inversely related to left ventricular ejection fraction (EF) and cardiac index (CI). ${ }^{9,11}$ ET-1 seems to be a key mediator in the pathogenesis of $\mathrm{PH}$ in chronic heart failure. ${ }^{9,11,12}$

The REACH-1 (Research on Endothelin Antagonism in Chronic Heart Failure) trial has used bosentan doses of $500 \mathrm{mg}$ daily in patients with advanced heart failure. The duration of the trial was planned for 24 weeks. Unfortunately, however, the REACH-1 trial has been terminated prematurely due to concerns about elevations in hepatic transaminases. ${ }^{13}$ Nevertheless, this study also demonstrated a significantly greater clinical improvement in patients who received bosentan therapy for 6 months compared to controls. We therefore administered bosentan in several of our patients with end-stage heart failure and $\mathrm{PH}$ as an off-label use during recent years. But we used a considerably lower daily bosentan dose than that used in the REACH-1 trial. We provide the results of a retrospective data analysis based on our medical records. We performed this analysis to assess whether low dose bosentan treatment is associated with improved pulmonary hemodynamics and clinical outcome in cardiac transplant candidates with $\mathrm{PH}$.

\section{Materials and Methods}

\section{Patients and study design}

Since January 2006, 183 patients with end-stage heart failure and longstanding $\mathrm{PH}$ were eligible for cardiac transplantation at our institution (-Fig. 1). All patients fulfilled the functional, echocardiographic, and hemodynamic indications for cardiac transplantation provided by the German Standing Committee on Organ Transplantation. ${ }^{8}$ However, all patients also had values of PAP $>35 \mathrm{mmHg}$, PVR $>240$ dyn $\times \mathrm{s} \times$ $\mathrm{cm}^{-5}$, and/or TPG $>15 \mathrm{mmHg}$ despite optimal treatment with angiotensin-converting enzyme-inhibitors, $\beta$-blockers, anticoagulants, digitalis derivatives or/and diuretics, ${ }^{1}$ whereas PAP and PVR decreased with the administration of intravenous phosphodiesterase III inhibitor. As mentioned before, elevated PAP, PVR, and/or TPG values are considered a contraindication for cardiac transplantation. ${ }^{8}$ To achieve transplantability, we decided to treat the aforementioned group of patients with oral bosentan as off-label use. Generally, all 183 patients with $\mathrm{PH}$ and end-stage heart failure were eligible for the off-label use of oral bosentan. However, initial bosentan administration was done under intensive care unit monitoring, including Swan-Ganz catheter measurement. Since

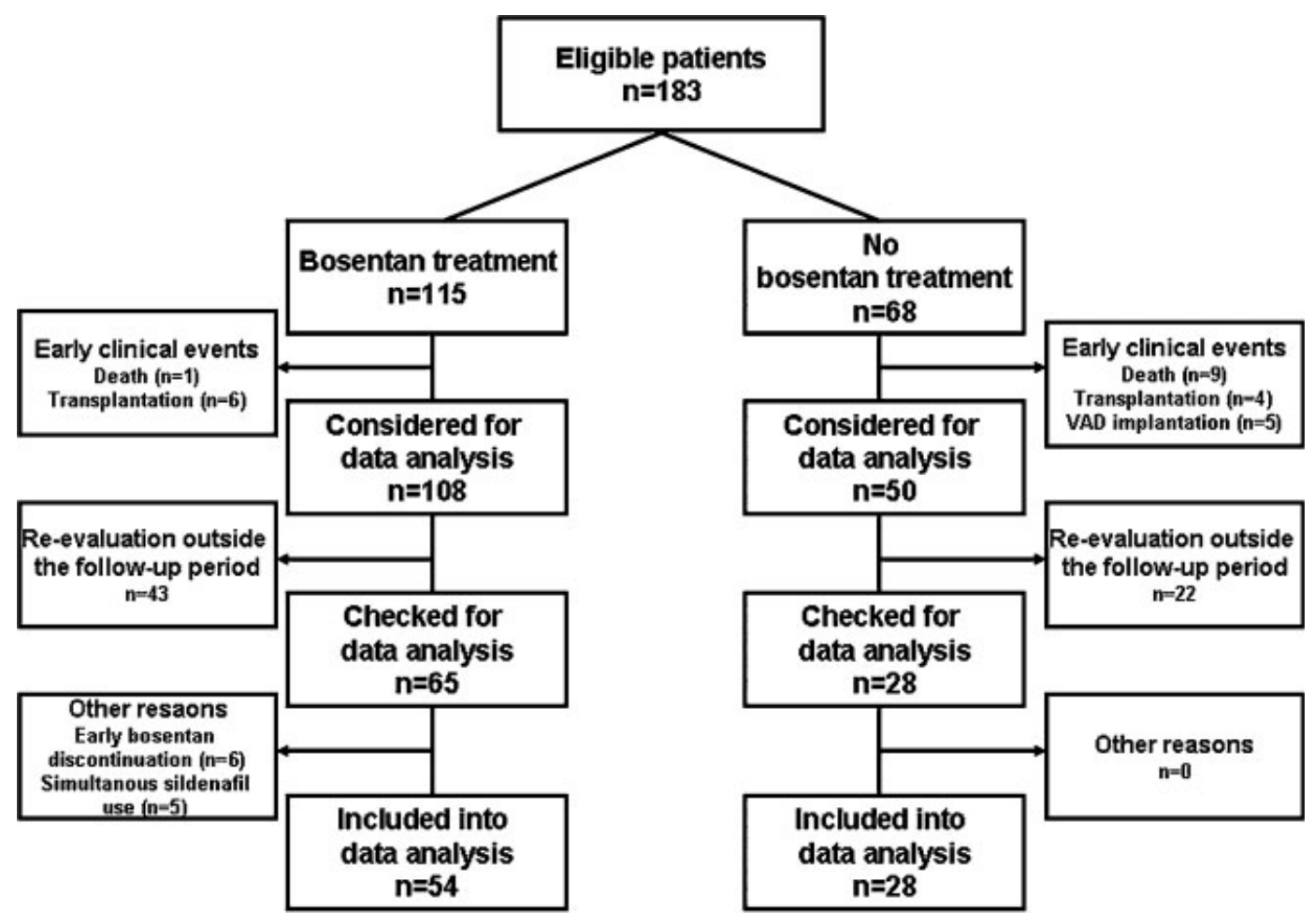

Figure 1 Study flow chart. 
intensive care unit capacity was limited, only 115 patients received bosentan. Sixty-eight other patients did not receive bosentan. The decision for bosentan administration was made by the head of the transplant unit, based on inclusion criteria and according to bed capacity. All patients gave their written informed consent to receive bosentan. The majority of patients started with an oral bosentan dose of $62.5 \mathrm{mg}$ bid ( $n=3$ with $32.5 \mathrm{mg}$ bid, $n=8$ with $125 \mathrm{mg}$ bid) and were uptitrated to $125 \mathrm{mg}$ bid. We performed a retrospective data analysis of clinical outcome parameters in patients who received bosentan or did not receive bosentan. For data analysis, we checked only those patients who had at least three echocardiographic and hemodynamic measurements within a time interval of 15 months. Patients with simultaneous sildenafil or iloprost treatment were excluded from data analysis. We finally included only those patients who received bosentan treatment or did not receive bosentan treatment during the entire follow-up period. Thus, we could retrospectively analyze the data of 54 patients receiving bosentan (BOS group) and of 28 patients not receiving bosentan (CON group). The latter group served as the control group. Patients were maintained on $125 \mathrm{mg}$ bid bosentan until the end of the study, cardiac transplantation, or death. The CON group received standard conventional medication. In both groups, echocardiographic parameters and hemodynamics were assessed at baseline, e.g., before bosentan treatment $\left(t_{0}\right)$, after 4 months ( $t_{1}$; range 2-6 months), and after 12 months ( $t_{2}$; range $9-15$ months). All measurements were performed as routine examinations at regular time intervals. Hemodynamics were measured using a Swan-Ganz pulmonary artery thermodilution catheter through the right internal jugular vein. Cardiac output (CO) was determined in triplicate and calculated by the Stewart-Hamilton indicator dilution formula. ${ }^{14}$ Cardiac index (CI) was calculated by $\mathrm{CO}$, taking current body surface into account. Central venous pressure (CVD), RV pressure, PAP, and PCWP were measured directly by right heart catheterization. Systemic vascular resistance (SVR), PVR and TPG were calculated according to standard formulas:

$$
\begin{aligned}
& \text { SVR }=[(\text { PAP-PCWP }) \times 80] / C I \\
& P V R=[(\text { PAP-PCWP }) \times 80] / C O \\
& T P G=P A P-P C W P
\end{aligned}
$$

Transthoracic echocardiography was performed to assess left ventricular end-diastolic and end-systolic diameter, right ventricular shortening, and left ventricular ejection fraction (Agilent Sonos 5500 device, Hewlett Packard, Andover, MA, USA). Since bosentan shows liver toxicity, ${ }^{15}$ biochemical safety parameters were also measured. In addition, we assessed clinical symptoms such as nocturia and dyspnea by questionnaire and peripheral edema by physical examination. We also registered clinical outcome parameters such as ventricular assist device (VAD) implantation, cardiac transplantation, and death in both groups up until June 2009.

\section{Statistical analysis}

We report categorical variables using the percentage of observations and express continuous variables as mean val- ues and standard deviation or median and interquartile range as appropriate. We tested normal distribution of the data using the Kolmogorov-Smirnov test. Normal distribution was considered present if $p$ values were above 0.05 . For comparative analyses between groups, we used Fisher's exact test, McNemar test, unpaired $t$-test, and Mann-Whitney test as appropriate. We used ANOVA and the Friedman test for evaluations of time-dependent effects. A $p$ value $<0.05$ (two-tailed test) was considered significant.

We generated Kaplan-Meier estimates to investigate the association between bosentan and survival probability during follow-up as a function of time after study inclusion. Logrank test was used to test for differences in survival rates between groups. We then examined the associations between bosentan and mortality risk using Cox proportional hazard analysis. Since treatment assignment was not based on random allocation and the bosentan and control group were therefore not expected to be completely comparable with regard to important covariates, we also analyzed whether propensity score adjustments were necessary. We used 38 baseline demographic, anthropometric, clinical, and biochemical variables for each patient, using logistic regression. The propensity score ranged from a low of 0.13 to a high of 0.63 The discriminate power of the propensity score was quantified by measurement of the receiver operating characteristics area and was found to be 0.651 only (95\% confidence interval: $0.465-0.836 ; p=0.110$ ), indicating that the two study groups were comparable with respect to baseline and clinical characteristics. We used the statistical software package SPSS, version 18 (SPSS, Inc., Chicago, IL, USA) to perform the analysis.

\section{Results}

Baseline characteristics of the study groups are given in - Table 1. Patients did not differ with regard to age, sex, and anthropometric data. Moreover, the prevalence of smokers and concomitant diagnoses such as diabetes, hypertension, elevated cholesterol levels, hyperuricemia, and dyspnea was similar between groups. Likewise, concomitant medications did not differ between groups. The majority of patients in each study group were in NYHA functional class III. The BOS group was, however, significantly younger than the CON group and suffered less often from diagnoses other than dilated cardiomyopathy or ischemic heart disease. More patients in the BOS group had pacemaker implants than in the CON group. In addition, some hemodynamic parameters such as right ventricular pressure, PAP, and PCWP were significantly higher in the BOS group than in the CON group at baseline (-Table 2 ). In the BOS group, the cut-off values for cardiac transplantation of PAP ( $>40 \mathrm{mmHg}$ ), PVR ( $>240$ dyn $\left.\times \mathrm{s} \times \mathrm{cm}^{-5}\right)$, and TPG $(>15)$ were exceeded in $75.9 \%, 67.9 \%$, and $47.2 \%$ of patients, respectively. The corresponding values for the CON group were $57.1 \%, 75.0 \%$, and $25.0 \%$, respectively.

In the BOS group, the median daily bosentan dose was $125 \mathrm{mg}$ (interquartile range: $125-125 \mathrm{mg}$; mean \pm SD: $139 \pm$ $50 \mathrm{mg} / \mathrm{d}$ ) at the beginning and $250 \mathrm{mg}$ (interquartile range: 250-250 mg; mean \pm SD: $216 \pm 77 \mathrm{mg} / \mathrm{d}$ ) at the end of the 
Table 1 Baseline characteristics of the bosentan and control group.

\begin{tabular}{|c|c|c|c|}
\hline Parameter & Bosentan group $(n=54)$ & Control group $(n=28)$ & $p$ value \\
\hline Age (years) & $54 \pm 11.0$ & $59.2 \pm 9.3$ & 0.015 \\
\hline Height $(\mathrm{cm})$ & $176 \pm 9$ & $174 \pm 9$ & 0.269 \\
\hline Weight $(\mathrm{kg})$ & $82.0 \pm 16.2$ & $80.4 \pm 14.0$ & 0.665 \\
\hline Body mass index $\left(\mathrm{kg} / \mathrm{m}^{2}\right)$ & $26.4 \pm 4.2$ & $25.8 \pm 5.9$ & 0.587 \\
\hline Gender (\% males) & 87.0 & 86.2 & $>0.999$ \\
\hline Heart rate (per min) & $77.4 \pm 13.4$ & $75.2 \pm 11.0$ & 0.472 \\
\hline Mean blood pressure $(\mathrm{mmHg})$ & $78.4 \pm 12.1$ & $80.2 \pm 12.8$ & 0.528 \\
\hline NYHA functional class $\geq \mathrm{III}(\%)$ & 79.63 & 86.21 & 0.380 \\
\hline \multicolumn{4}{|l|}{ Diagnosis } \\
\hline Dilated cardiomyopathy (\%) & 46.3 & 34.5 & 0.351 \\
\hline Coronary heart disease (\%) & 44.4 & 27.6 & 0.240 \\
\hline Others $^{\mathrm{a}}(\%)$ & 9.3 & 37.9 & 0.008 \\
\hline \multicolumn{4}{|l|}{ Concomitant diagnoses } \\
\hline Diabetes mellitus (\%) & 35.2 & 28.6 & 0.625 \\
\hline Hypercholesterolemia (\%) & 9.3 & 14.3 & 0.483 \\
\hline Hyperuricemia (\%) & 9.3 & 14.3 & 0.483 \\
\hline Chronic kidney disease (\%) & 11.1 & 28.6 & 0.064 \\
\hline Hypertension (\%) & 33.3 & 21.4 & 0.314 \\
\hline Nicotine abuse (\%) & 13.0 & 21.4 & 0.351 \\
\hline \multicolumn{4}{|l|}{ Pacemaker and defibrillator } \\
\hline Only pacemaker implantation (\%) & 57.4 & 32.1 & $<0.001$ \\
\hline Only defibrillator implantation (\%) & 0 & 0 & $>0.999$ \\
\hline Implantation of both (\%) & 7.4 & 7.1 & $>0.999$ \\
\hline \multicolumn{4}{|l|}{ Medications } \\
\hline ACE inhibitors/AT blockers (\%) & 72.2 & 82.1 & 0.420 \\
\hline$\beta$-blockers (\%) & 79.6 & 71.4 & 0.420 \\
\hline Diuretics (\%) & 88.9 & 78.6 & 0.320 \\
\hline Digitalis derivatives (\%) & 42.6 & 25.0 & 0.150 \\
\hline Oral anticoagulants (\%) & 66.7 & 46.4 & 0.098 \\
\hline Aspirin (\%) & 39.3 & 24.0 & 0.201 \\
\hline
\end{tabular}

${ }^{a}$ Amyloidosis, cor pulmonale, hypertrophic cardiomyopathy, condition after coronary artery bypass grafting

follow-up period. Down titration of bosentan was necessary in 6 patients due to marked decreases in PAP and PVR. In 2 of these 6 patients, a parallel increase in aspartate aminotransferase and alanine aminotransferase occurred, whereas in 1 of the 6 patients only alanine aminotransferase increased.

In the CON group, hemodynamic and echocardiographic parameters did not change significantly during follow-up (-Table 2). In the BOS group, echocardiographic parameters also remained unchanged. However, mean blood pressure and all measured hemodynamic parameters showed significant improvements in the BOS group during follow-up. The three determinants of PVR (i.e., PAP, PCWP, and CO) improved significantly from $t_{0}$ to $t_{2}$ by $18.0 \%, 15.7 \%$, and $23.5 \%$, respectively. Consequently, mean PVR decreased significantly from a mean of 382 dyn $\times \mathrm{s} \times \mathrm{cm}^{-5}$ to 256 dyn $\times \mathrm{s} \times \mathrm{cm}^{-5}$. In the BOS group, the percentage of patients with values below the cut-offs increased from $t_{0}$ to $t_{2}$ for PAP from $24.1 \%$ to $44.4 \%$ $(p=0.007)$, for PVR from $32.1 \%$ to $56.6 \%(p=0.013)$, and for TPG from $52.8 \%$ to $73.6 \%$ ( $p=0.007$ ). SVR values declined by $17.0 \%$. None of the patients in the BOS group developed hypotension (mean arterial pressure $<50 \mathrm{mmHg}$ ).

In the $\mathrm{CON}$ group, the percentages increased nonsignificantly from $42.9 \%$ to $53.6 \%$ for PAP ( $p=0.581$ ), from $25.0 \%$ to $39.3 \%$ for PVR ( $p=0.388)$, and remained constant for TPG ( $75.0 \%$ vs. $71.4 \%, p>0.999$ ). SVR values tended to decline to a similar extend compared with the BOS group. NYHA functional class did not change significantly during follow-up, neither in the BOS group nor in the CON group (data not shown).

Biochemical safety parameters are presented in - Table 3 . In the BOS group, mean concentrations of liver enzymes such 
Table 2 Hemodynamic and echocardiographic parameters of the bosentan (BOS) and control (CON) group at baseline and during follow-up.

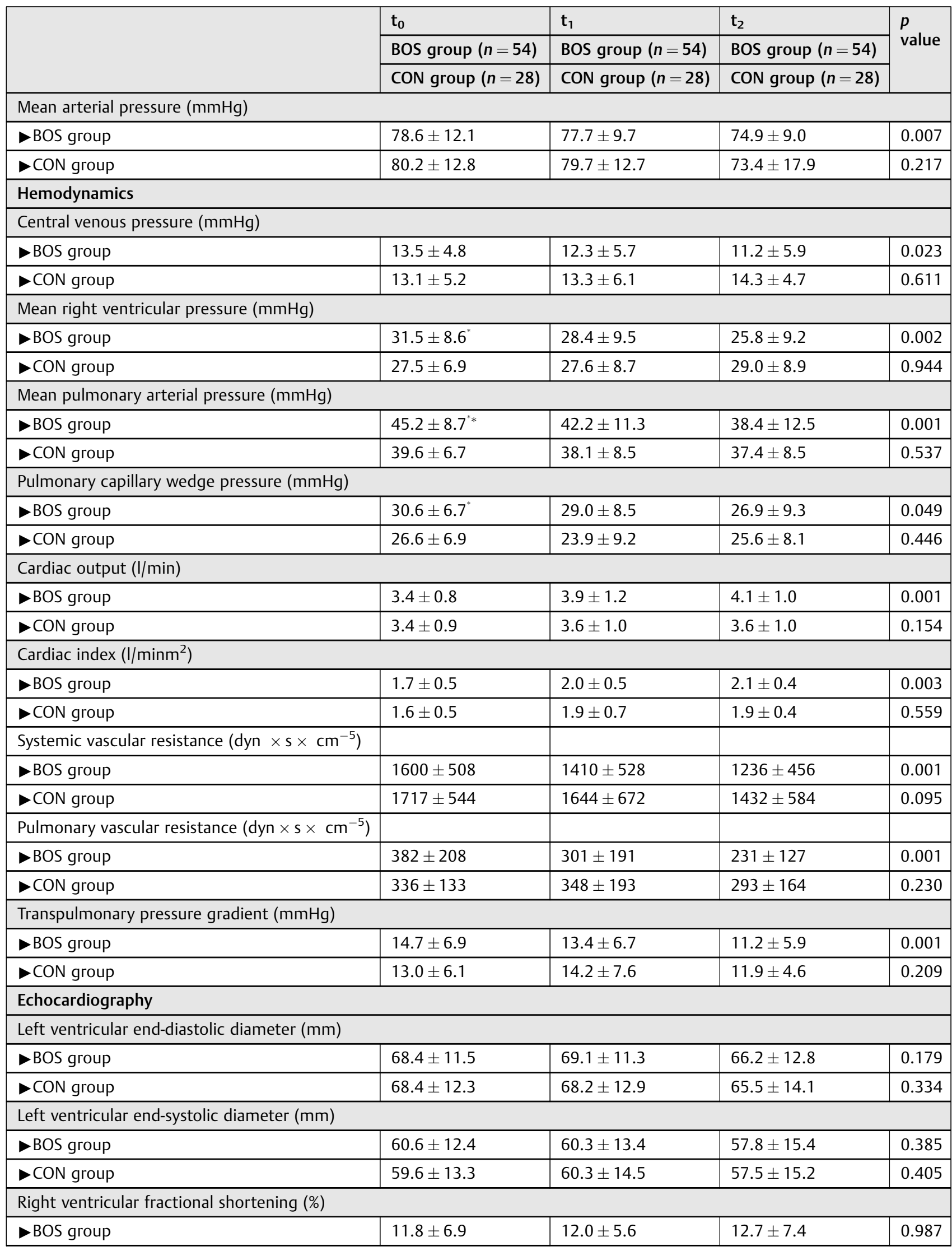


Table 2 (Continued)

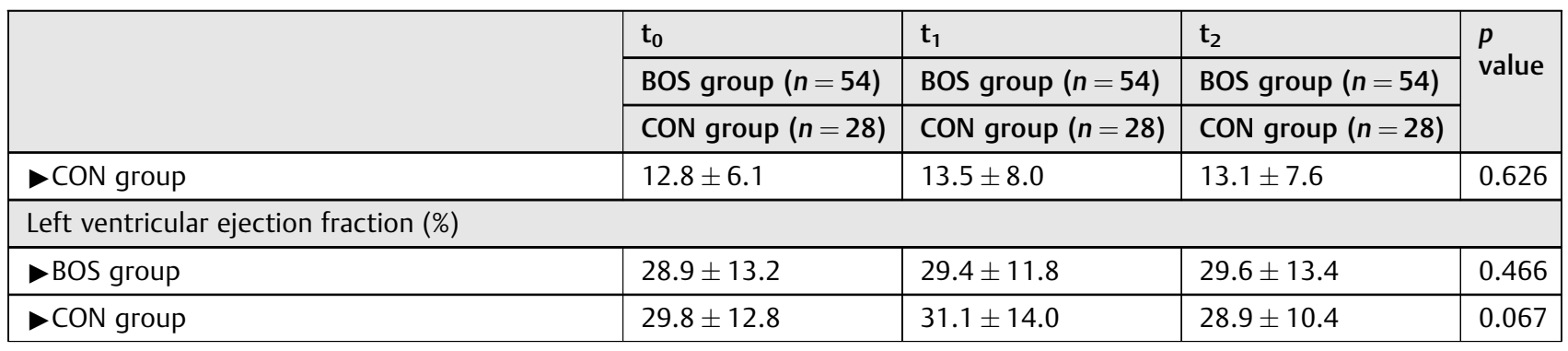

$\mathrm{t}_{0}$ : baseline; $\mathrm{t}_{1}: 4$ months after inclusion; $\mathrm{t}_{2}: 12$ months after inclusion; ${ }^{*}, " * p<0.05$ and $p<0.01$ vs. controls at the same time point

as aspartate aminotransferase, alanine aminotransferase, and gamma-glutamyltransferase remained constant. In the BOS group, there was a decrease in hematocrit values, which also tended to occur in the CON group. Sodium concentrations improved significantly in the BOS group from $t_{0}$ to $t_{2}$.

In the BOS group, 35 patients (64.8\%) were not hospitalized, whereas 15 patients (27.8\%), one patient (1.9\%), and three patients (5.6\%) were hospitalized once, twice, and three times, respectively, during follow-up. The corresponding values for the CON group were 20 (71.4\%), 8 (28.6\%), zero, and zero, respectively. In the BOS and CON group, the percentage of patients with nocturia $(22.2 \%$ vs. $28.6 \%$, respectively), peripheral edema ( $20.4 \%$ vs. $14.4 \%$, respectively), and dyspnea ( $18.5 \%$ vs. $7.1 \%$, respectively) did not differ significantly ( $p=0.205-0.592)$.

During follow-up, ten patients in the BOS group (18.5\%) and one patient in the CON group (3.6\%) were transplanted $(p=0.088)$. All transplanted patients were alive at the end of the follow-up period. One patient in the BOS group and 11 patients in the CON group died during follow-up. Causes of death were acute lung edema in the BOS group, and multiple organ failure $(6 \times)$, low output syndrome $(2 \times)$, intracranial bleeding $(1 \times)$, intestinal ischemia $(1 \times)$, and pancytopenia $(1$ $x)$ in the CON group. - Fig. 2 illustrates the Kaplan-Meier survival curves on the waiting list after $t_{2}$ according to study group. One-year survival on the waiting list was $93.3 \%$ in the BOS group and $70.7 \%$ in the CON group $(p=0.020)$. The corresponding values for freedom from cardiac related deaths was $93.3 \%$ and $100 \%$, respectively ( $p=0.407$ ).

In the univariate Cox regression analysis, bosentan treatment was significantly related to reduced risk of mortality on the waiting list $(\mathrm{RR}=0.109 ; 95 \% \mathrm{CI}: 0.013-0.881 ; p=0.038)$. Bosentan treatment remained an independent predictor of reduced mortality risk on the waiting list after propensity score adjustment $(\mathrm{RR}=0.107 ; 95 \% \mathrm{CI}$ : 0.013-0.869; $p=$ 0.036).

\section{Discussion}

Our data demonstrate that patients with end-stage heart failure and $\mathrm{PH}$ not receiving bosentan have a poor prognosis.
Results support earlier findings of high mortality rates in heart failure patients with $\mathrm{PH}^{7}$. However, this study could also demonstrate significantly higher survival rates of endstage heart failure patients with $\mathrm{PH}$ who were treated with bosentan compared to patients who were not treated with bosentan while awaiting cardiac transplantation. Moreover, compared with the patients not treated with bosentan a higher percentage of bosentan-treated patients tended to be transplanted during follow-up.

Although the REACH-1 trial was stopped prematurely due to liver toxicity, this earlier study already demonstrated a significantly greater clinical improvement in patients with advanced heart failure who received bosentan therapy for 6 months. $^{13}$ Thus, our data support the assumption that bosentan may be a new treatment option for end-stage heart failure patients. Compared to the REACH-1 trial, our retrospective data analysis is based on a longer duration of followup and a sicker patient population. In addition, our analysis includes invasive hemodynamic assessment, and defined hemodynamic criteria including TPG for enrolment.

The development of new and more effective pharmacological treatment strategies for $\mathrm{PH}$ in patients with end-stage heart failure is urgently needed. In this context, bosentan seems to be a promising candidate. In our analysis, bosentan treatment was associated with an improvement in various hemodynamic parameters. For example, PVR decreased by $33 \%$ in the BOS group, whereas it declined only nonsignificantly by $12.8 \%$ in the CON group (-Table 2 ). Our results are in line with the fact that bosentan treatment is also able to reduce PVR in patients with pulmonary arterial hypertension. ${ }^{16,17}$ In addition, our data analysis demonstrates an improvement in RV pressure, which is an important prognostic value for survival in patients with chronic heart failure. ${ }^{4}$ The improvement in RV pressure and the partial revision in $\mathrm{PH}$ may therefore be an important explanation for the fact that one year after $t_{2}$ the survival rate on the waiting list was $22 \%$ higher ( $93 \%$ vs. $71 \%$ ) in the BOS group than in the CON group. Note that patients with end-stage heart failure are at an increased risk for cardiac decompensation. This can lead to systemic immunosuppression and thus to an increased risk of developing multiple organ failure. 
32 Bosentan Effects on Hemodynamics Hefke et al.

Table 3 Biochemical parameters of the bosentan and control group at baseline and during follow-up.

\begin{tabular}{|c|c|c|c|c|}
\hline & $t_{0}$ & $t_{1}$ & $t_{2}$ & \multirow[t]{3}{*}{$p$ value } \\
\hline & BOS group $(n=54)$ & BOS group $(n=54)$ & BOS group $(n=54)$ & \\
\hline & CON group $(n=28)$ & CON group $(n=28)$ & CON group $(n=28)$ & \\
\hline \multicolumn{5}{|l|}{ Liver function } \\
\hline \multicolumn{5}{|c|}{ Aspartate aminotransferase (U/I) } \\
\hline BOS group & $38.7 \pm 37.0$ & $28.22 \pm 8.76$ & $28.2 \pm 8.8$ & 0.319 \\
\hline- CON group & $28.0 \pm 9.1$ & $29.2 \pm 10.4$ & $31.4 \pm 12.5$ & 0.471 \\
\hline \multicolumn{5}{|c|}{ Alanine aminotransferase (U/I) } \\
\hline BOS group & $54.4 \pm 12.4$ & $25.9 \pm 14.0$ & $26.4 \pm 15.1$ & 0.093 \\
\hline -CON group & $33.4 \pm 18.2$ & $28.3 \pm 14.6$ & $26.7 \pm 36.9$ & 0.042 \\
\hline \multicolumn{5}{|c|}{ gamma-glutamyltransferase (U/I) } \\
\hline BOS group & $114 \pm 88$ & $103 \pm 88$ & $112 \pm 99$ & 0.048 \\
\hline -CON group & $150 \pm 137$ & $144 \pm 124$ & $190 \pm 182$ & 0.303 \\
\hline \multicolumn{5}{|c|}{ Total bilirubin (mg/dL) } \\
\hline BOS group & $1.30 \pm 0.86$ & $0.92 \pm 0.53$ & $0.88 \pm 0.52$ & 0.163 \\
\hline -CON group & $0.97 \pm 0.48$ & $1.02 \pm 0.66$ & $1.15 \pm 0.89$ & 0.608 \\
\hline \multicolumn{5}{|c|}{ Kidney function } \\
\hline \multicolumn{5}{|c|}{ Creatinine $(\mathrm{mg} / \mathrm{dL})$} \\
\hline BOS group & $1.39 \pm 0.4$ & $1.45 \pm 0.51$ & $1.67 \pm 0.80$ & 0.080 \\
\hline - CON group & $1.39 \pm 0.4$ & $1.43 \pm 0.44$ & $1.51 \pm 0.48$ & 0.519 \\
\hline \multicolumn{5}{|c|}{ Blood urea nitrogen (mg/dL) } \\
\hline BOS group & $68.2 \pm 36.4$ & $75 \pm 47$ & $85.0 \pm 56.9$ & 0.093 \\
\hline- CON group & $76.2 \pm 43.6$ & $82 \pm 54$ & $84.6 \pm 50.0$ & 0.639 \\
\hline \multicolumn{5}{|l|}{ Hematology } \\
\hline \multicolumn{5}{|c|}{ Leukocytes $\left(10^{9} / \mathrm{I}\right)$} \\
\hline -BOS group & $8.97 \pm 12.5$ & $9.15 \pm 12.4$ & $8.92 \pm 12.7$ & 0.722 \\
\hline- CON group & $8.41 \pm 3.16$ & $8.44 \pm 2.86$ & $8.20 \pm 2.29$ & 0.857 \\
\hline \multicolumn{5}{|c|}{ Red blood cells $\left(10^{12} / \mathrm{I}\right)$} \\
\hline BOS group & $4.33 \pm 0.63$ & $4.23 \pm 0.66$ & $4.17 \pm 4.12$ & 0.106 \\
\hline CON group & $4.63 \pm 1.9$ & $4.24 \pm 0.64$ & $4.12 \pm 0.83$ & 0.435 \\
\hline \multicolumn{5}{|c|}{ Hemoglobin (mg/dL) } \\
\hline BOS group & $12.5 \pm 1.9$ & $12.6 \pm 2.1$ & $12.5 \pm 3.13$ & 0.215 \\
\hline -CON group & $14.1 \pm 5.7$ & $13.0 \pm 2.53$ & $12.7 \pm 4.1$ & 0.025 \\
\hline \multicolumn{5}{|l|}{ Hematocrit (\%) } \\
\hline BOS group & $38.1 \pm 5.2$ & $37.2 \pm 6.1$ & $36.4 \pm 5.18$ & 0.005 \\
\hline -CON group & $40.4 \pm 11.8$ & $38.3 \pm 7.6$ & $37.8 \pm 12.7$ & 0.063 \\
\hline \multicolumn{5}{|l|}{ Platelets $\left(10^{9} / \mathrm{I}\right)$} \\
\hline BOS group & $197 \pm 61$ & $212 \pm 72$ & $212 \pm 78$ & 0.145 \\
\hline- CON group & $198 \pm 81$ & $204 \pm 84$ & $217 \pm 107$ & 0.476 \\
\hline \multicolumn{5}{|l|}{ Electrolytes } \\
\hline \multicolumn{5}{|c|}{ Sodium (mmol/l) } \\
\hline - BOS group & $137 \pm 4$ & $136 \pm 4$ & $138 \pm 4$ & 0.008 \\
\hline -CON group & $137 \pm 6$ & $136 \pm 5$ & $137 \pm 5$ & 0.513 \\
\hline
\end{tabular}


Table 3 (Continued)

\begin{tabular}{|c|c|c|c|c|}
\hline & $t_{0}$ & $t_{1}$ & $t_{2}$ & $p$ value \\
\hline & BOS group $(n=54)$ & BOS group $(n=54)$ & BOS group $(n=54)$ & \\
\hline & CON group $(n=28)$ & CON group $(n=28)$ & CON group $(n=28)$ & \\
\hline - BOS group & $4.25 \pm 0.50$ & $4.08 \pm 0.54$ & $4.08 \pm 0.54$ & 0.611 \\
\hline$>$ CON group & $4.05 \pm 0.76$ & $4.09 \pm 0.80$ & $4.00 \pm 0.51$ & 0.889 \\
\hline
\end{tabular}

$t_{0}$ : baseline; $t_{1}: 4$ months after inclusion; $t_{2}: 12$ months after inclusion

In our analysis, bosentan treatment was associated with a more pronounced reduction in PVR than in SVR. Similar results have been observed in patients with chronic heart failure and high circulating ET-1 concentrations who received short-term intravenous bosentan. ${ }^{10}$ Likewise, our data of unchanged echocardiographic measurements in the BOS group confirm an earlier multicenter randomized controlled trial (RCT) of bosentan in heart failure patients with severe systolic dysfunction and $\mathrm{PH} .{ }^{18}$ In that earlier RCT, echocardiographic measurements were not influenced by bosentan treatment.

Since bosentan is an ET-1 receptor antagonist, our data support the assumption that ET-1 plays an important role in the etiology of $\mathrm{PH}$ in heart failure patients. This assumption is in line with the fact that the plasma concentrations of ET-1 are increased in patients with heart failure and correlate with the prognosis. ${ }^{9,11}$ In animal models of heart failure, ET-1 antagonists are also able to improve hemodynamics and survival. $^{19}$

In addition to the improvement in the survival of patients on the waiting list, bosentan seems to offer an important additional benefit. Due to the significant decrease in hemodynamic parameters, a relatively high number of patients in the BOS group compared to the CON group could be transplanted during follow-up (18.5\% vs. $3.6 \%$ ). Since all patients had end-stage heart failure, cardiac transplantation was the

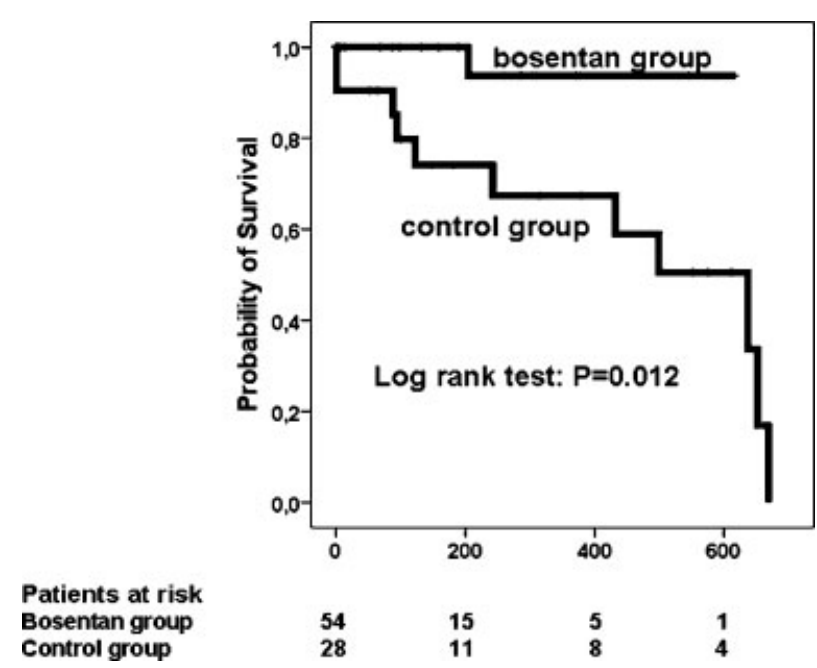

Figure 2 Probability of survival on the waiting list after $t_{2}$ in the bosentan group and the control group. last option for these patients. None of the transplanted patients had died by the end of the follow-up period, supporting the assumption that survival after cardiac transplantation seems to be similar in patients without pre-existing $\mathrm{PH}$ and in patients where preoperatively existing $\mathrm{PH}$ is reversible. ${ }^{7}$

Elevated liver aminotransferase levels seemed to be characteristic for bosentan treatment. ${ }^{9,16}$ In the present data analysis, however, the BOS group in its entirety showed no pathological elevations in serum alanine aminotransferase or aspartate aminotransferase concentrations from $t_{0}$ to $t_{2}$. This is probably due to the fact that we prescribed only $250 \mathrm{mg}$ bosentan daily, whereas others have used daily doses of $500 \mathrm{mg}$. ${ }^{4}$ In previous investigations, peripheral edema occurred more frequently in patients treated with bosentan. ${ }^{9}$ In our retrospective analysis, clinical complications such as edema and dyspnea as well as the percentage of patients who reported nocturemia were not adversely affected by bosentan treatment. In addition, the hospitalization rate was similar between the BOS and CON groups.

In contrast to earlier results, ${ }^{16}$ the BOS group showed no improvement in NYHA functional class. However, it should be noted that NYHA functional class is a subjective parameter. Hemodynamic parameters are much more reliable for the assessment of cardiac function. Initial $\mathrm{CI}$ measurements demonstrate that our patients suffered from advanced heart failure, leaving only a limited possibility of improvement in NYHA functional class.

It must be stated that our data analysis has several limitations due to its retrospective nature. It lacks randomization, blinding, and a clear study protocol. In addition, this retrospective data analysis is short of defined dose up- and down-titration, follow-up visits, and adverse event reporting. Although we have performed propensity score adjustments to assess mortality rates, this can not compensate for nonrandomization. Finally, the study is a single center report without an independent core lab, adjudicated event committee, data monitoring and safety committee. Since earlier randomized controlled trials with bosentan focused on patients with pulmonary arterial hypertension, ${ }^{16,17}$ a randomized controlled multicenter study with survival on the waiting list as the primary endpoint and cardiac transplantation as a secondary endpoint is urgently needed.

In conclusion, our data demonstrate that bosentan treatment is associated with improvements in hemodynamics and clinical outcome in end-stage heart failure patients with PH. If 
these results are confirmed by randomized controlled trials bosentan may represent a treatment option.

\section{References}

1 Galiè N, Hoeper MM, Humbert M, et al. Guidelines for the diagnosis and treatment of pulmonary hypertension. Eur Heart J 2009;30; 2493-2537

2 Moreas DL, Colucci WS, Givertz MM. Secondary pulmonary hypertension in chronic heart failure-The role of the endothelium in pathophysiology and management. Circulation 2000;102;17181723

3 Grigioni F, Potena L, Galiè N, et al. Prognostic implications of serial assessments of pulmonary hypertension in severe chronic heart failure. J Heart Lung Transplant 2006;25;1241-1246

4 Mylona P, Cleland JGF. Update of REACH- 1 and MERIT-HF clinical trials in heart failure. Eur J Heart Fail 1999;1;197-200

5 Mehra MR, Kobashigawa J, Starling R, et al. Listing criteria for heart transplantation: international society for heart and lung transplantation guidelines for the care of cardiac transplant candidates2006. J Heart Lung Transplant 2006;25;1024-1042

6 Addonizio LJ, Gersony WM, Robbins RC, et al. Elevated pulmonary vascular resistance and cardiac transplantation. Circulation 1987;76;V52-V55

7 Gude E, Simonsen S, Geiran OR, et al. Pulmonary hypertension in heart transplantation: discrepant prognostic impact of pre-operative compared with 1-year post-operative right heart hemodynamics. J Heart Lung Transplant 2010;29;216-223

8 Hoppe J-D, Schreiber H-L. Richtlinien zur Organtransplantation gemäß Â§ 16 Transplantationsgesetz. Dtsch Arztebl 2000;97;A396-A-411

9 Cowburn PJ, Cleland JGF. Endothelin antagonists for chronic heart failure: do they have a role? Eur Heart J 2001;22;1772-1784
10 Kiowski W, Sütsch G, Hunziker P, et al. Evidence for endothelin-1mediated vasoconstriction in severe chronic heart failure. Lancet 1995;346;732-736

11 Pacher R, Stanek M, Hülsmann M, et al. Prognostic impact of big endothelin-1 plasma concentrations compared with invasive hemodynamic evaluation in severe heart failure. J Amer Coll Cardiol 1996;27;633-641

12 Schulz U, Tenderich G, Schulte-Eistrup S, et al. Bosentan in pulmonary hyertension secondary to left heart failure. Curr Med Res Opin 2007;23;S77-S82

13 Ghio S, Gavazzi A, Campana C, et al. Independent and additive prognostic value of right ventricular systolic function and pulmonary artery pressure in patients with chronic heart failure. J Am Coll Cardiol 2001;37;183-188

14 Mathews L. Paradigm shift in hemodynamic monitoring. The Internet Journal of Anesthesiology 2007;11;2

15 Humbert M, Segal ES, Kiely DG, Carlsen J, Schwierin B, Hoeper MM. Result of European post-marketing surveillance of bosentan in pulmonary hypertension. Eur Respir J 2007;30;338-344

16 Galiè N, Rubin LJ, Hoeper MM, et al. Treatment of patients with mildly symptomatic pulmonary arterial hypertension with bosen$\tan$ (EARLY study): a double-blind, randomised controlled trail. Lancet 2008;371;2093-2100

17 Rubin LJ, Badesch DB, Barst RJ, et al. Bosentan therapy for pulmonary arterial hypertension. N Engl J Med 2002;346;896-903

18 Kaluski E, Cotter G, Leitman M, et al. Clinical and hemodynamic effects of bosentan dose optimization in symptomatic heart failure patients with severe systolic dysfunction, associated with secondary pulmonary hypertension-a multi-center randomized study. Cardiology 2008;109;273-280

19 Moreas DL, Colucci WS, Givertz MM. Secondary pulmonary hypertension in chronic heart failure-The role of the endothelium in pathophysiology and management. Circulation 2000;102; $1718-1723$ 